\title{
Low Energy Muons as Probes of Thin Films and Surfaces
}

\author{
E. Morenzoni \\ Paul Scherrer Institut, Villigen, Switzerland
}

Received March 7, 1997; revised May 14, 1997

\begin{abstract}
Polarized muons with kinetic energies of a few eV (epithermal $\mu^{+}$) can be generated by slowing down energetic muons in appropriate moderators consisting of a thin layer of a van der Waals gas frozen on a substrate. The availability of polarized muons with kinetic energies in the eV to several $\mathrm{keV}$ range opens the possibility to extend the $\mu \mathrm{SR}$ technique to the study of thin films and surfaces (low energy $\mu \mathrm{SR}, \mathrm{LE}-\mu \mathrm{SR}$ ). We summarize the characteristics of the very slow polarized muons and of a low energy beam based on the moderation technique. We discuss the implantation of muons in thin film samples and the potential and limitations of LE- $\mu$ SR. As an example first results obtained by implanting slow $\mu^{+}$in a sample consisting of a $\mathrm{Ni}$ film deposited on $\mathrm{Ag}$ are presented.
\end{abstract}

\section{Introduction}

The $\mu$ SR technique, although younger than most of the other nuclear solid state techniques, has reached in the last decade a remarkable level of maturity. This is demonstrated in the present issue by the diversity of the methods encompassed and by the wide spectrum of questions addressed by it. Nevertheless, as with all fields of science, new methods have to be continuously developed because of changing challenges in understanding the current objects of investigations. The state of the art of a method such as $\mu$ SR which relies on accelerators and beam lines is heavily dependent on the quality and characteristics of the available muon beams. A new promising technique under development is what we will call here low energy $\mu \mathrm{SR}$ (LE- $\mu \mathrm{SR}$ ), i.e., the generation and use of muons with kinetic energies between a few $\mathrm{eV}$ and a few tens of $\mathrm{keV}$ for muon spin research studies.

Until now, available beams for $\mu$ SR studies have delivered polarized muons with energies in the $\mathrm{MeV}$ range. These energetic muons penetrate deeply into the material under investigation (typically a few $100 \mu \mathrm{m}$ ) and come to rest over a wide region of the sample. Therefore, they essentially probe bulk properties and no investigations as a function of the implantation depth or sample thickness are possible. Questions concerning thin films, surfaces and interfaces are generally 
not accessible by conventional $\mu \mathrm{SR}$ techniques. For this polarized muons with variable energy between $\sim 10 \mathrm{eV}$ and a few tens of $\mathrm{keV}$ are required. By varying the energy in this interval, implantation depths between typically fractions of a $\mathrm{nm}$ and a few hundred $\mathrm{nm}$ can be obtained. With the availability of such particles thin films, solid-solid, and vacuum-solid interfaces become accessible to $\mu \mathrm{SR}$ studies thus offering a new investigative tool to these growing fields and greatly extending the field of application of $\mu \mathrm{SR}$.

In this paper we give a short overview of the present status and prospects of LE- $\mu$ SR with particular emphasis on the LE- $\mu$ SR project at the Paul Scherrer Institute (PSI). We are at the beginnings of LE- $\mu$ SR and the study of methodological aspects specific to LE- $\mu \mathrm{SR}$ is still predominant. The generation of slow muons, a low energetic muon beam, and the characteristics of epithermal muons are presented in Sections 2-4. The interaction of the slow muons with samples is discussed in Section 5. In Section 6 we will give an example of LE- $\mu$ SR and try to realistically assess the opportunities offered by the very slow $\mu^{+}$.

\section{Generation of Low Energy $\mu^{+}$Beams}

Compared to the case of other less exotic particles, for the production of low energetic $\mu^{+}$one is faced with more arduous initial conditions, which are determined by the physics of the muon generation. Muons originate from decaying pions and are therefore born energetic. In the pion rest frame they have a kinetic energy of $\sim 4.1 \mathrm{MeV}$, which is the energy of the so-called surface muons (100\% polarized) [1]. Pions and muons form a secondary beam (the primary beam consisting of the protons impinging on the production target). The phase space properties of a typical $\mu^{+}$beam are quite poor with beam spots of several $\mathrm{cm}^{2}$ and divergencies of $\sim 100 \mathrm{mrad}$. In principle, a very simple way to lower the energy of charged particles is to degrade them in a low $Z$ material, but this broadens the energy spectrum. Multiple scattering further reduces the quality and the intensity of the beam to unacceptable levels. This is what is obtained by tuning a surface $\mu^{+}$beam to momenta much lower than $29 \mathrm{MeV} / \mathrm{c}$ (corresponding to $4.1 \mathrm{MeV}$ energy); the intensity decreases with momentum as $p^{3.5}$ and this method provides reasonable beams only down to several hundreds of $\mathrm{keV}$ of kinetic energy. In the past, various methods have been proposed to produce low energy beams with good phase space quality and small energy spread (for an overview see [2]), but only two of them bear relevance for LE- $\mu$ SR. They are based on a source of thermal or epithermal muons which are electrostatically accelerated to the desired energy.

The first technique, under development at the KEK Laboratory (Japan), uses the fact that a fraction of $\mu^{+}$stopped in an appropriate material such as a hot tungsten foil are reemitted from the surface as muonium at thermal energy. These atoms are resonantly ionized by intense pulsed lasers synchronized with the muonium emission by the accelerator pulse, yielding the source of thermal muons. 
To maximize the yield the foil is arranged directly in the $500 \mathrm{MeV}$ proton beam together with the primary target producing the pions and muons [3].

The second method is technologically simpler and best suited for the continuous beams at PSI. It is based on the moderation technique where surface muons from a secondary beam line are slowed down in a layer of a van der Waals bound solid $[4,5]$. In these weakly bound, wide band gap materials, a nonnegligible fraction of muons do not completely thermalize and are emitted as free epithermal particles even if the surface has a positive work function.

\section{The Very Slow Muon Beam at PSI}

The principle of the moderation method is shown in Fig. 1. Surface $\mu^{+}$impinge on the moderator from the substrate side. The beam is formed by collecting at the downstream side of the moderator the very slow $\mu^{+}$escaping from a thin layer of a condensed van der Waals gas. It should be noted that the essential physical processes take place in this condensed layer and that the substrate, where about $50 \%$ of the beam is stopped, acts only as a predecelerator. The general layout of the apparatus is shown in Fig. 2. We give here only a succinct description for better understanding and refer to previous publications $[4,6]$ for details. The set-up is contained in a single ultra-high vacuum (UHV) chamber with a base vacuum of $10^{-10}$ mbar. Ultra-high vacuum conditions are essential for optimal and stable moderator conditions and are also a prerequisite for most of the proposed experiments in surface and thin-film physics.

In Fig. 2 three main components are recognizable: the moderator, the electrostatic acceleration and transport section, and the set-up for LE- $\mu$ SR studies. The mod-

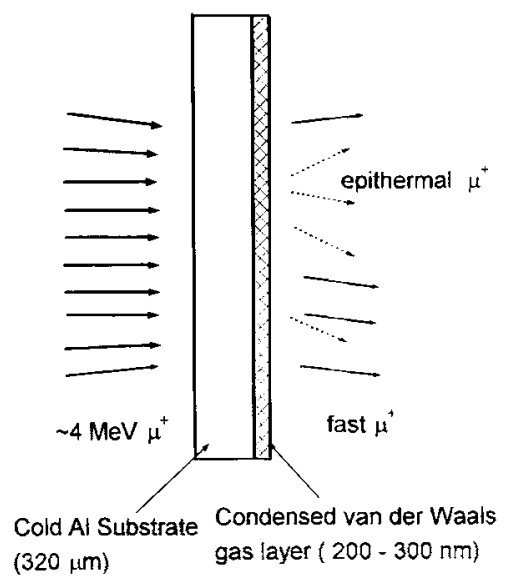

Fig. 1. Principle of the moderation technique for the production of very slow polarized $\mu^{+}$. The total thickness of the moderator is chosen to maximize the yield of epithermal muons. 


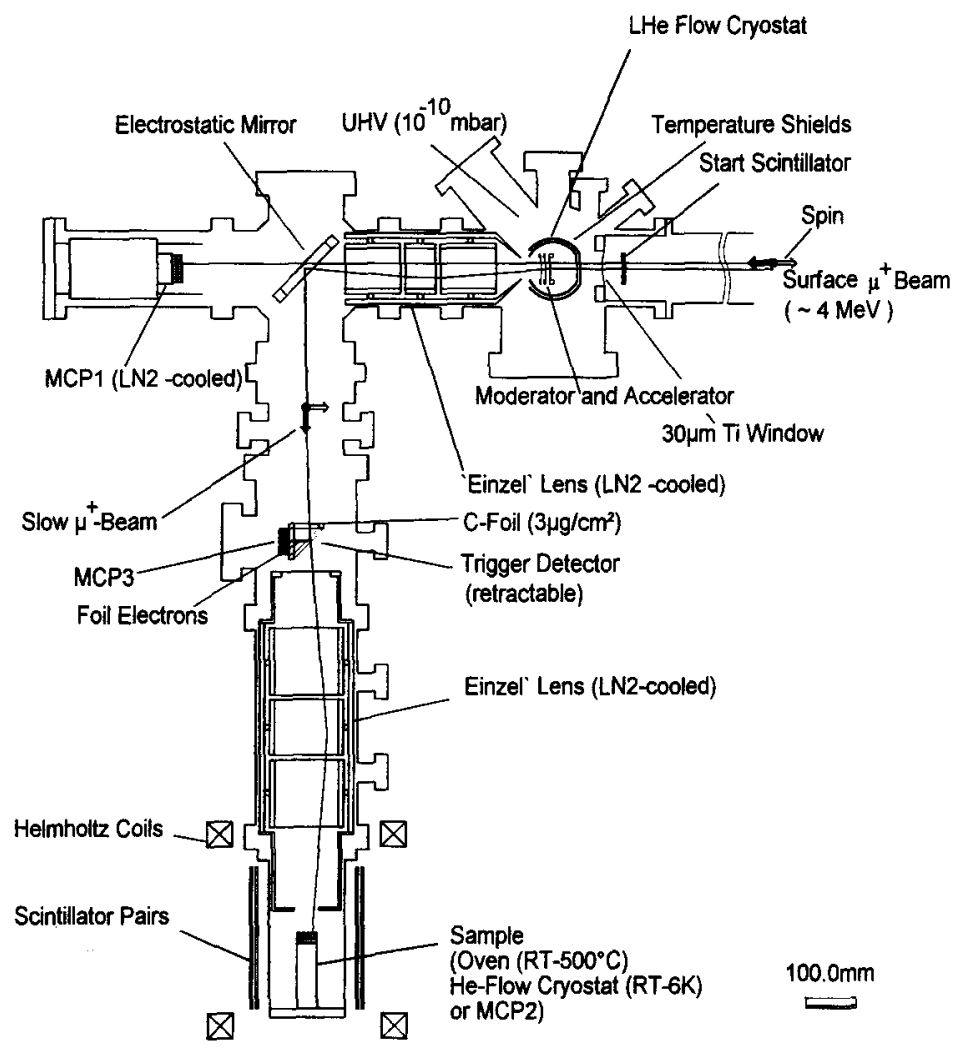

Fig. 2. Apparatus for low energy $\mu \mathrm{SR}$ at the Paul Scherrer Institute.

erator is obtained by condensing a van der Waals gas (generally $\mathrm{Ar}$ or $\mathrm{N}_{2}$ ) on an Al substrate cooled by a liquid helium flow cryostat. The substrate is electrically insulated and can be raised to a voltage as high as $20 \mathrm{kV}$. The very slow $\mu^{+}$extracted from the production region enter the transport section. Two "einzel" lenses and an electrostatic mirror are used to focus and deflect the very slow $\mu^{+}$onto the sample. The mirror separates the slow muons from the dominant fraction of nonmoderated energetic $\mu^{+}$. Depending on the experimental requirements various holders are available, which allow sample temperatures between 6 and $800 \mathrm{~K}$.

The transport section accommodates an essential element for LE- $\mu S R$, namely the so-called trigger detector which is used to provide the start signal of time differential $\mu$ SR measurements. It consists of a very thin carbon foil (thickness $\sim 3$ $\mu \mathrm{g} / \mathrm{cm}^{2}$, corresponding to only $\sim 70$ atomic layers) combined with a microchannel plate detector located perpendicularly to the foil (MCP 3 in Fig. 2). It is based on the detection of electrons which, ejected by the $\mu^{+}$traversing the foil, are directed by a small system of grids to the MCP where they are detected. The very thin 


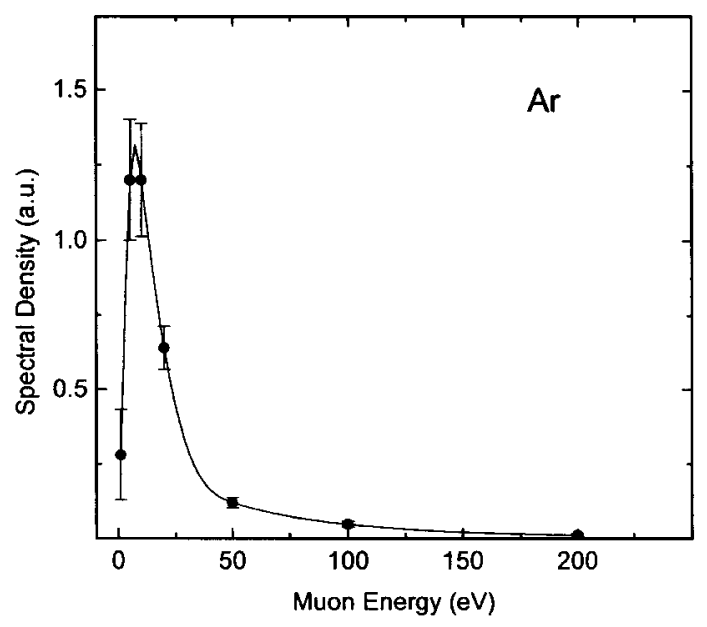

Fig. 3. Energy distribution of epithermal $\mu^{+}$emitted from solid Ar.

foil results in a minimal influence on the $\mu^{+}$trajectory, while allowing an efficient $(\sim 70 \%)$ and fast (intrinsic timing resolution $\leq 1 \mathrm{~ns})$ detection. Since the initial energy spread of the epithermal muons is small (see Section 4 and Fig. 3), the energy width of the $\mu^{+}$beam is determined by the energy straggling introduced by the interaction with the carbon foil. The resulting uncertainty (typically $\sim 400$ $\mathrm{eV}$ ) does not affect the stop distribution of $\mu^{+}$with a kinetic energy of several $\mathrm{keV}$, whereas at lower energies its effect cannot be neglected [7]. It can be reduced, for instance, by introducing a pulsed cylindrical electrode placed after the trigger detector. By using the timing signal from this detector as a trigger and choosing an appropriate pulse shape, a sizable reduction of the energy spread could be achieved.

\section{Physics and Characteristics of Moderated Muons}

The generation of very slow muons relies on the specific behavior of charged particles in condensed van der Waals gases, particularly in the energy regime preceding thermalization. Briefly, $\mu^{+}$implanted at $\mathrm{MeV}$ energies in the moderator rapidly lose energy by electronic collisions, in the beginning mainly by ionization and excitation then, as the energy drops, by electron capture (muonium formation) and electron loss (muonium break-up). Because of the wide energy gap, these electronic processes are forbidden or strongly suppressed for those $\mu^{+}$ that have lowered their energy to epithermal energies inside the frozen gas layer. As a consequence, in the final stage before thermalization, the slowing down is completely dominated by soft muon-atom elastic collisions and these muons are likely not to thermalize but escape from the frozen layer at epithermal energies. The transition from stronger to weaker energy loss mechanisms seems peculiar 
to van der Waals condensed gases. It leads to large escape depths and particular efficient moderation to $\mathrm{eV}$ energies especially in those elements with large band gap energy such as $\mathrm{Ne}, \mathrm{Ar}$, and $\mathrm{N}_{2}$. The relationship between reduced energyloss rate and large escape depth has been recently demonstrated in [6], where the escape depth $L$ of epithermal $\mu^{+}$from $\mathrm{Ar}$ and $\mathrm{Kr}$ was found to be $L=111 \pm 8$ $\mathrm{nm}$ and $L=47 \pm 9 \mathrm{~nm}$, respectively.

In our present set-up with a planar moderator, efficiencies to convert fast muons into very slow ones of the order of $10^{-4}-10^{-5}$ have been obtained. This is about two orders of magnitude larger than efficiencies obtained from single ionic crystal insulators or from metals. The moderation mechanism is also reflected in the energy distribution of the emitted muons. For solid Ar (Fig. 3) the distribution has a peak at $\sim 10 \mathrm{eV}$ (of the order of the band gap energy which is $\sim 14 \mathrm{eV}$ ) with a FWHM of $\sim 15 \mathrm{eV}$ and a tail to larger energies.

The fastness of the moderation process (slowing down time $\sim 10 \mathrm{ps}$ ) practically conserves the initial polarization of the incoming surface muons. This is an essential property for an unrestricted use of moderated muons as magnetic microprobes in thin-film and surface studies. Measurement of the polarization $P$ of the epithermal $\mu^{+}$yield for those emitted from solid $\operatorname{Ar} P=92 \pm 7.5 \%$, from solid $\mathrm{N}_{2} P=90 \pm 10 \%$ and from solid $\mathrm{Kr} P=90 \pm 10 \%$ [4].

The moderation efficiency can be optimized by choosing appropriate growth conditions of the solid gas layer (deposition rate and temperature, temperature cycling, heat treatment) [6]. A temperature of $\sim 15 \mathrm{~K}$ for $\mathrm{Ar}$ and of $\sim 30 \mathrm{~K}$ for $\mathrm{Kr}$ turns out to be optimal. For $\mathrm{Kr}$ and Ar films deposited at $\sim 10 \mathrm{~K}$ the efficiency increases after annealing. This behavior can be related to the morphological (granularity, porosity) and structural (defects, voids) characteristics of thin films of condensed gases which depend on the growth conditions. As a general rule, one can state that growth conditions leading to larger and less defective grains and to an open porous structure result in moderators of superior quality. The long term stability of the moderator, an important practical aspect for the operation of the very slow $\mu^{+}$beam, is ensured by UHV conditions. At a pressure of $10^{-10}$ mbar the moderation efficiency remains stable over a period of several days.

\section{Interaction of Low Energy Muons with Solids and Surfaces}

In order to fully exploit the potential of LE- $\mu$ SR various aspects of the interaction of muons in solids and surfaces such as implantation profiles, reemission, backscattering and neutralization at the surface must be better understood. In this respect, it is interesting to note that the understanding of the collision dynamics of muons in solids and at surfaces is also central to the generation of very slow polarized muons. Very few studies have been deserved to these aspects. Some guidance can be drawn from analogous proton interactions since, from the point of view of the collision mechanisms involved, the $\mu^{+}$can be treated as a light 
proton. However, also in this case there is very little experimental data for energies below $10 \mathrm{keV}$.

In complex systems (e.g., multilayers) a precise knowledge of the depth distribution may be needed for a well defined implantation in the sample and for the interpretation of the results. In other cases it is sufficient to characterize the distribution by their mean projected range $R_{\mathrm{p}}$, the projection of the path of the particle along the normal to the sample surface, and the width of the distribution by the variance $\Delta R_{\mathrm{p}}$. Various analytical treatments of the transport of particles in matter are available, but more realistic results are obtained by computer simulations based on Monte Carlo methods, where the slowing down and scattering of the projectile through the solid are followed step by step $[8,9]$.

A well known representative of this kind of approach is the program TRIM ("Transport of Ions in Matter") [8], which is used to calculate range distributions and backscattering coefficients for a variety of projectile/sample combinations. A recent comparison between experimental data on $R_{\mathrm{p}}$ and $\Delta R_{\mathrm{p}}$ of few $\mathrm{keV}$ protons with various simulations [10] has given good agreement with the predictions of a more sophisticated TRIM version [9], which extends the range of TRIM applicability to smaller energies. This program was used to calculate the range and range straggling of $\mu^{+}$in some samples (Eckstein W., private communication). Figure 4 presents the depth profile of $10 \mathrm{keV} \mu^{+}$in $\mathrm{Ni}$, showing the typical maximum and the tail to lower depths. Figure 5 shows the results for $R_{\mathrm{p}}$ and $\Delta R_{\mathrm{p}}$ of $\mu^{+}$in $\mathrm{SiO}_{2}, \mathrm{Ni}$, and $\mathrm{Au}$ as a function of the implantation energy. The figure illustrates the material dependence of the implantation profile. At fixed energy, decreasing $Z$ increases the mean penetration depth and narrows the relative width of the distribution. The penetration depends on electronic energy loss processes and on elastic scattering so that protons at the same

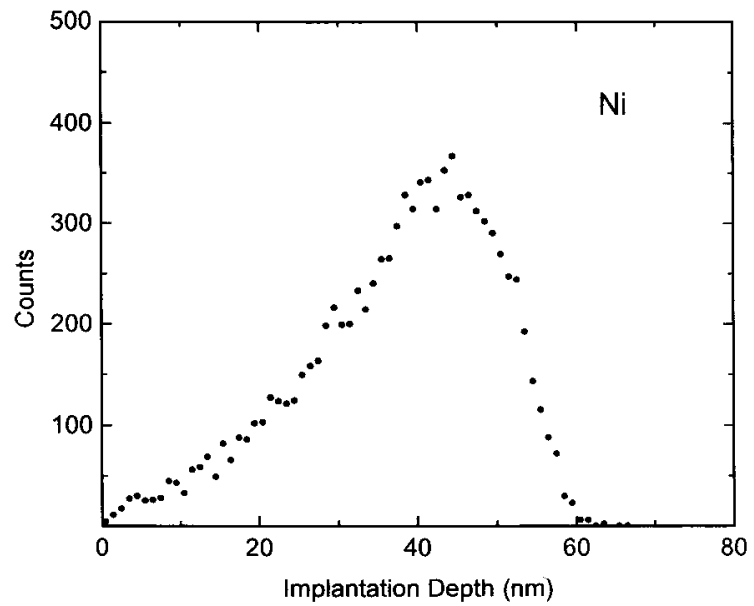

Fig. 4. Calculated depth distribution of $10 \mathrm{keV} \mu^{+}$implanted in $\mathrm{Ni}$ (Eckstein W., private communication). 


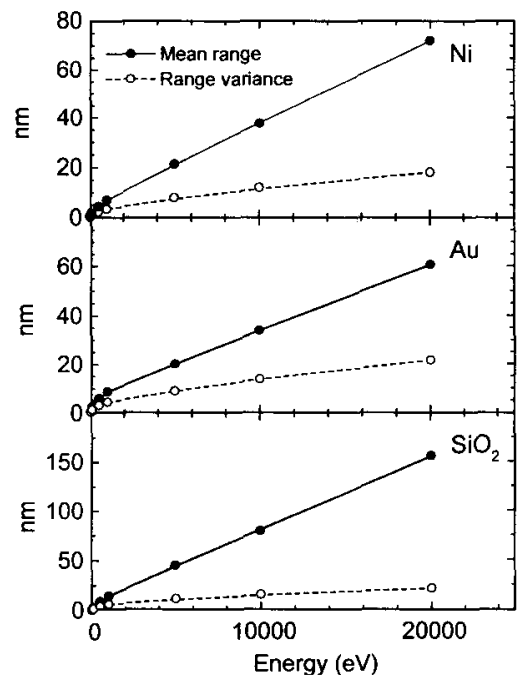

Fig. 5. Calculated energy dependence of the mean projected range and of the variance of the depth distribution of $\mu^{+}$in $\mathrm{Ni}, \mathrm{Au}$ and $\mathrm{SiO}_{2}$ samples (Eckstein $\mathrm{W}$., private communication).

energy penetrate deeper than muons and have a slightly larger depth distribution. It should be noted that diffusion and trapping of the $\mu^{+}$after thermalization may modify the stop distribution given by the electronic and elastic processes. Therefore, depending on the sample characteristics and experimental parameters, the thermal interactions must be also considered to determine the final $\mu^{+}$site distribution when they decay.

To achieve direct surface sensitivity, softlanding of the $\mu^{+}$is necessary. At energies in the $\mathrm{eV}$ range we can expect that the muon does not penetrate into most of the samples, but here again detailed investigations of the muon-surface interactions are necessary. Reflection at the surface cannot be neglected and we estimate that at $\sim 10 \mathrm{eV}$ about $60 \%$ of the $\mu^{+}$are reflected from a medium-mass sample [11]. Simultaneous neutralization may also occur which could be used to study the electronic state properties of the surface.

\section{Potential and Limitations of LE- $\mu$ SR}

The basic potential and limitations of very slow muons are similar to those of the more energetic ones discussed in the introductory chapter of this issue [1]. They are determined by the intrinsic properties of the muon which make $\mu$ SR spectroscopy most powerful in the field of small moments magnetism, short range or random magnetic order and in the use of muonium as a hydrogen isotope. The essential new features are depth resolution and accessibility to the wide new fields of investigations offered by thin films, interfaces, and surfaces. Therefore, 
we discuss here only the specific implications of LE- $\mu$ SR. For various reasons experimental conditions for LE- $\mu$ SR are more stringent, and the low to very low energy of the muons requires special considerations for the design of the set-up and for an optimum implantation into the sample. The lowest energies in the $\mathrm{eV}$ range are the most demanding since, in this case, the muons must be decelerated just in front of the sample and reflection must be taken into account. A UHV environment is required not only for the generation of the very slow $\mu^{+}$ but also for their application to thin films and surface investigations. No window can be tolerated in front of the sample; a $15 \mathrm{keV} \mu^{+}$stops in a $130 \mathrm{~nm}$ thick $\mathrm{C}$ foil. Good and reproducible sample quality necessitates in-situ preparation and characterization or appropriate transfer devices and this has to fit to the specific requirements of $\mu$ SR spectrometry as positron counters and magnetic fields close to the sample. Accessible magnetic field ranges are more limited in LE- $\mu$ SR since stray fields of coils generating several $\mathrm{kG}$ magnetic fields may deflect the very slow muons. A magnetic shielding or compensating coils for critical parts of the apparatus (as for instance the trigger detector) are necessary in these cases.

The accuracy of the entrance time of the muon into the sample is determined by the energy straggling of the muons in the $\mathrm{C}$ foil of the trigger detector and by the distance of the detector from the sample. This translates into a time uncertainty of $\sim 6 \mathrm{~ns}$ ( $\mathrm{rms}$ ) but can be reduced by increasing the preacceleration voltage. Frequency resolution and time window do not differ from conventional $\mu S R$. Concerning beam intensities, a large difference exists between conventional $\mu$ SR and LE- $\mu$ SR. Generally for a typical $\mu$ SR measurement one collects $\sim 10^{6}$ decay positrons in a spectrum and $\mu \mathrm{SR}$ is not an intensity limited technique. At the moment this is not the case for LE- $\mu \mathrm{SR}$. The very slow polarized muon beam at PSI, presently the most intense in the world, allows a detected event rate of $\sim 15 \mathrm{~Hz}$ (defined as the rate of detected decay $\mathrm{e}^{+}$originating from slow $\mu^{+}$ implanted in the sample). This figure includes all efficiencies and solid angles. The signal-to-noise ratio is very good. This implies acquisition times between a few hours and a few days, a routine situation for many fields of science but not for $\mu \mathrm{SR}$ where sometime a measurement can be completed in minutes. Various developments (improvements of the transport system, new target geometry, use of higher intensity secondary $\mu^{+}$beam) not requiring extraordinary investments are underway with single improvement factors between 2 and 10 . Nevertheless, the present intensity already allows investigations of thin film samples. An example of the potential is shown in Fig. 6 presenting preliminary results (Birke M., Glückler H., Hofer A., Litterst J., Morenzoni E., Niedermayer Ch., Schatz A., Schatz G., unpubl.). In this experiment $15 \mathrm{keV} \mu^{+}$were implanted in a sample made of $30 \mathrm{~nm} \mathrm{Ni}$ sputtered on $\mathrm{Ag}$. At this energy the muons stop in the $\mathrm{Ag}$ layer at a mean distance of $\sim 30 \mathrm{~nm}$ from the Ni/Ag interface. The transverse field measurement at a temperature above the Curie temperature of Ni $\left(T_{\mathrm{c}}=631\right.$ $\mathrm{K})$ shows the muons precessing without depolarization. When we lower the temperature below $T_{\mathrm{c}}$ the precession signal is damped, a manifestation of temperature dependent depolarization. The depolarization (damping) increases with de- 


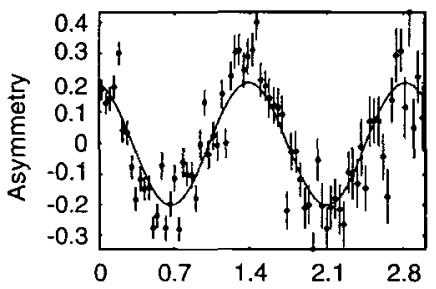

a

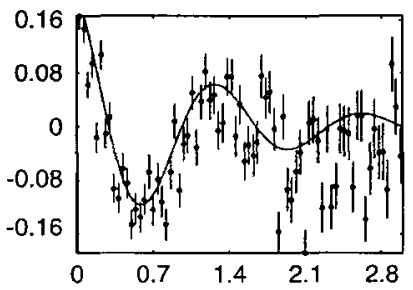

b
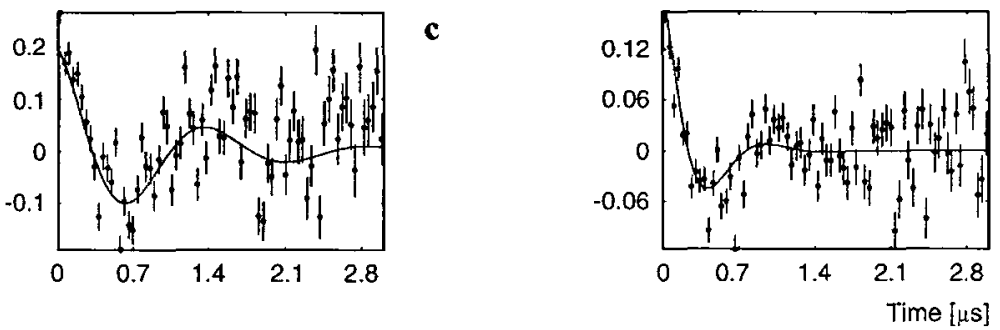

Fig. 6. Asymmetry signal from the decay of $15 \mathrm{keV}$ muons stopped in a Ni/Ag sample and precessing in a $5 \mathrm{mT}$ transverse magnetic field. a $T=683 \mathrm{~K}$, b $T=613 \mathrm{~K}$, c $T=603 \mathrm{~K}$, d $T=293 \mathrm{~K}$.

creasing temperature and follows the magnetization curve of the thin $\mathrm{Ni}$ film. This is an indication of the penetration of the magnetic field originating inside the Ni-film in the Ag substrate. The result shows the potential of the use of very slow polarized $\mu^{+}$for the study of small magnetic fields and couplings in thin samples and multilayers.

We can expect that the routine exploitation of this technique will increase as the source will develop in strength. The sophistication of the experiments will also develop parallel to the investments in the very slow muon beam and in the instrumentation for sample preparation and characterization.

\section{Acknowledgements}

I thank all the collaborators who over the years have contributed to the developments presented in this paper and whose names can be found in $[4,6]$. I also thank W. Eckstein for calculating the implantation profiles for muons with his program.

\section{References}

[1] Roduner E.: Appl. Magn. Reson. 13, 1 (1997)

[2] Simons L., Morenzoni E., Kottmann F. in: Exotic Atoms in Condensed Matter (Benedek G., Schneuwly H., eds.). Springer Proceedings in Physics 59, 33 (1992)

[3] Nagamine K., Miyake Y., Shimomura K., Birrer P., Marango J.P., Iwasaki M., Strasser P., Kuga T.: Phys. Rev. Lett. 74, 4811 (1995)

[4] Morenzoni E., Kottmann F., Maden D., Matthias B., Meyberg M., Prokscha Th., Wutzke Th., Zimmermann U.: Phys. Rev. Lett. 72, 2793 (1994) 
[5] Harshman D.R., Mills A.P., Beveridge J.L., Kendall K.R., Morris G.D., Senba M., Warren J.B., Rupaal A.S., Turner J.H.: Phys. Rev. B 36, 8850 (1987)

[6] Morenzoni E., Prokscha Th., Hofer A., Matthias B., Meyberg M., Wutzke Th., Birke M., Glückler H., Litterst J., Niedermayer Ch., Schatz G.: J. Appl. Phys. (1997), in press.

[7] Morenzoni E., Birke M., Glückler H., Hofer A., Litterst J., Meyberg M., Niedermayer C., Prokscha Th., Schatz G., Wutzke Th.: Hyp. Int. (1997), in press.

[8] Ziegler J.F., Biersack J.P., Littmark U.: The Stopping and Range of Ions in Solids, vol. 1. NewYork: Pergamon Press 1985.

[9] Eckstein W.: Computer Simulation of Ion-Solid Interactions. Berlin: Springer 1991.

[10] Mayer M., Eckstein W.: Nucl. Instrum. Methods B 94, 22 (1994)

[11] Thomas E.W., Janev R.K., Smith J.: Nucl. Instrum. Methods B 69, 427 (1992)

Author's address: Dr. Elvezio Morenzoni, Paul Scherrer Institut, CH-5232 Villigen PSI, Switzerland 